\title{
Intractable Coronary Spasm Requiring Percutaneous Coronary Intervention after Coronary Artery Bypass Grafting in a Patient with Moyamoya Disease
}

\author{
Hyeon A Kim, M.D., Young Su Kim, M.D., Wook Sung Kim, M.D., Ph.D. \\ Department of Thoracic and Cardiovascular Surgery, Samsung Medical Center, Sungkyunkwan University School of Medicine, Seoul, Korea
}

\section{ARTICLE INFO}

Received May 20, 2020

Revised August 11, 2020

Accepted August 12, 2020

Corresponding author

Wook Sung Kim

Tel 82-2-3410-2213

Fax 82-2-3410-0089

E-mail wooksung.kim@samsung.com

ORCID

https://orcid.org/0000-0001-7808-3385

\begin{abstract}
Moyamoya disease (MMD) is characterized by progressive steno-occlusive lesions of the distal or proximal branch of the internal carotid arteries, and cerebrovascular symptoms are its major complications. Extracranial vascular involvement including the coronary artery has been reported, and some case reports have described variant angina or myocardial infarction. However, no report has yet described a case of myocardial infarction after coronary artery bypass grafting (CABG). Here, we present a patient with MMD who suffered cardiac arrest caused by myocardial infarction due to a coronary spasm after offpump CABG and who was discharged successfully after treatment with a veno-arterial extracorporeal membrane oxygenator and percutaneous coronary intervention.
\end{abstract}

Keywords: Moyamoya disease, Coronary vasospasm, Coronary artery disease, Coronary artery bypass surgery

\section{Case report}

We describe the case of a 47-year-old woman who was admitted to another center with recurrent syncope events, where she was diagnosed with moyamoya disease (MMD) based on brain magnetic resonance angiography and transfemoral cerebral angiography findings (Fig. 1). To rule out a cardiogenic cause of recurrent syncope events, echocardiography was conducted, which revealed mild left ventricular (LV) dysfunction, an LV ejection fraction of $40 \%$, and apical regional wall motion abnormalities. Two-vessel disease was revealed by coronary angiography (CAG), after which the patient was referred to Samsung Medical Center for coronary artery bypass grafting (CABG) surgery.

When we reviewed the CAG results, we identified chronic total occlusion lesions in the left anterior descending artery (LAD), 90\% stenosis of the proximal left circumferential artery, and 50\% stenosis in the right coronary artery (RCA) (Fig. 2).

We performed off-pump CABG. The left internal thoracic artery was grafted onto the LAD and the right internal thoracic artery to the ramus intermedius and obtuse
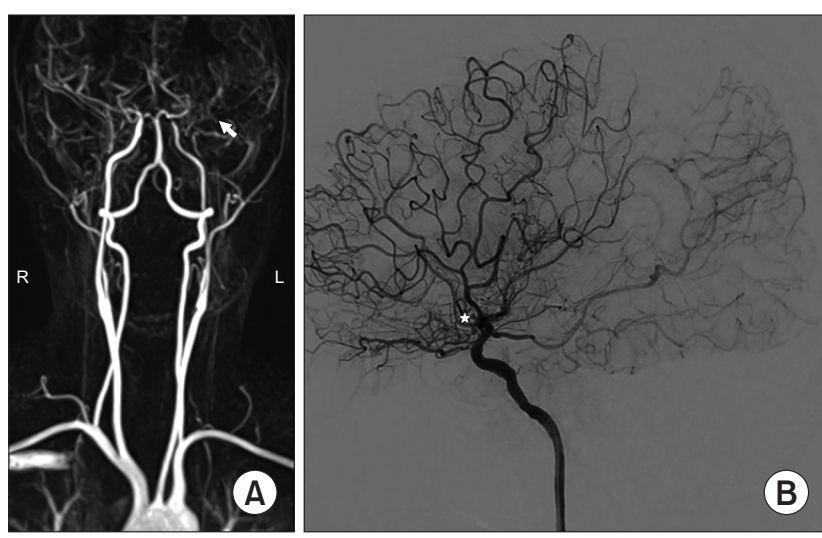

Fig. 1. Preoperative brain magnetic resonance angiography and transfemoral cerebral angiography. (A) Steno-occlusive lesion of the left distal internal carotid artery (white arrow). (B) At the distal part of the steno-occlusive lesion, there are areas of collateral circulation like a puff of smoke (white star).

marginal. Following our routine protocol, papaverine wrapping for the graft vessels was done. Intraoperatively, there were no significant events such as profound hypotension (refractory to volume loading or increments of medi- 

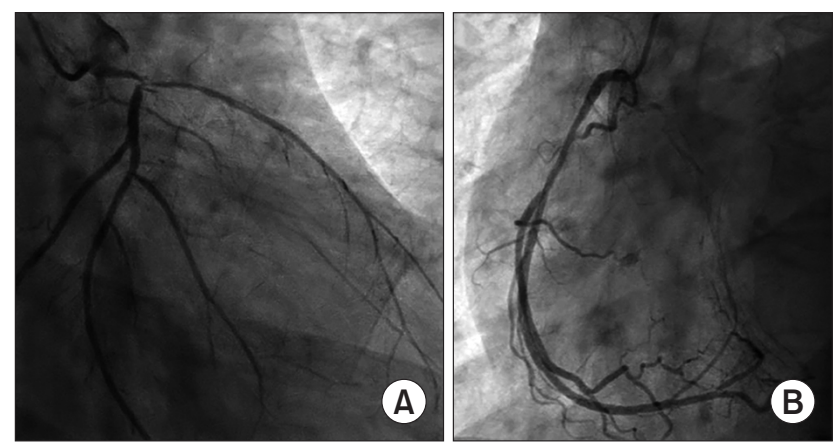

Fig. 2. Preoperative coronary angiography of unstable angina with 2 -vessel disease. (A) Chronic total occlusive lesion in the left anterior descending artery and proximal $90 \%$ stenosis of the left circumflex artery. (B) A 50\% stenosis lesion of the right coronary artery.

cation doses) or metabolic acidosis. During the operation, the total estimated blood loss was $1,500 \mathrm{~mL}$ and total input volume was more than $3,500 \mathrm{~mL}$.

After surgery, the patient was immediately admitted to the cardiac intensive care unit (ICU). The initial vital signs were as follows: systemic blood pressure, 90/62 $\mathrm{mm} \mathrm{Hg}$; heart rate, 72 beats per minute; and normal sinus rhythm under continuous intravenous infusion of norepinephrine $(0.15 \mu \mathrm{g} / \mathrm{kg} / \mathrm{min})$ and dopamine $(3 \mu \mathrm{g} / \mathrm{kg} / \mathrm{min})$.

One hour after ICU admission, significant ST depression was observed on electrocardiography. Profound hypotension developed soon after, requiring incremental infusion of inotropes, with continuous intravenous administration of epinephrine $(0.1 \mu \mathrm{g} / \mathrm{kg} / \mathrm{min})$, norepinephrine $(0.1 \mu \mathrm{g} / \mathrm{kg} /$ $\mathrm{min})$, dopamine $(3 \mu \mathrm{g} / \mathrm{kg} / \mathrm{min})$, and nitroglycerine $(0.5 \mu \mathrm{g} /$ $\mathrm{kg} / \mathrm{min}$ ). Two hours after ICU admission, the patient's vital signs improved and the inotrope requirements decreased. Three hours after ICU admission, the patient exhibited pulseless electrical activity, and we immediately began cardiopulmonary resuscitation. During cardiopulmonary resuscitation, a peripheral veno-arterial extracorporeal membrane oxygenator (ECMO) was inserted, and the patient was moved to the CAG room for evaluation.

Although, the graft vessels had good flow with no stenosis, newly appeared diffuse vasospastic lesions were present in all 3 native coronary arteries. Intracoronary nitroglycerin injection was sufficient to relieve the vasospastic lesions of the LAD and left circumferential artery, but not sufficient to relieve the RCA lesion. Despite repeated intracoronary nitroglycerin and nicorandil injections, the vasospastic lesion of the mid-RCA was not relieved with thrombolysis, under conditions of myocardial infarction grade 1 distal flow. Therefore, the attending cardiologist made the decision to perform stent insertion in the vasospastic lesion of
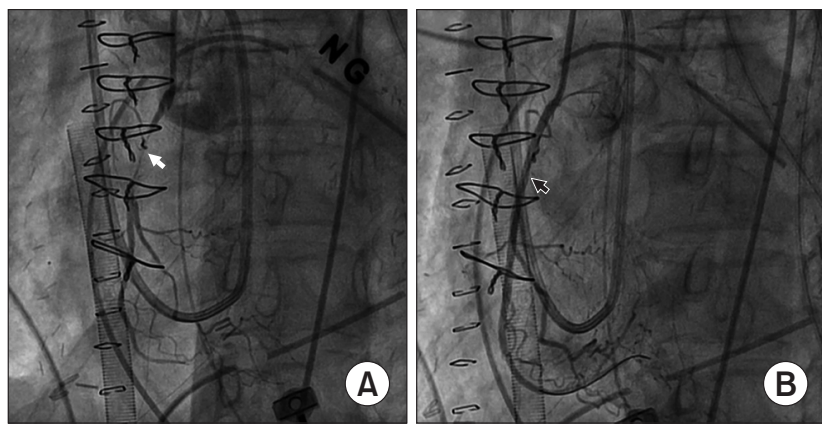

Fig. 3. Coronary artery angiography after extracorporeal membrane oxygenator insertion. (A) An intractable vasospastic lesion in the right coronary artery not relieved by repeated intracoronary nitroglycerin injections (white arrow). (B) Successful percutaneous coronary intervention with stent implantation in the right coronary artery (black arrow).

\section{the RCA (Fig. 3).}

On postoperative day (POD) 3, follow-up echocardiography revealed that the LV ejection fraction was $40 \%$ and that there were no new regional wall motion abnormalities. At this time point, the patient's vital signs were relatively stable and the requirements for inotropes had declined. Three days after CABG surgery, surgical removal of ECMO and open vascular repair were successfully performed. On POD 13, further follow-up echocardiography showed improved LV systolic function, with an ejection fraction of $55 \%$ and improved wall motion in the apical walls of the LV.

During hospitalization, the patient had to be administered antibiotics for 2 weeks because of a catheter-related infection. The patient was discharged on POD 26 without surgery-related complications. After discharge with dual anti-platelet therapy (aspirin and ticagrelor), calcium-channel blockers (CCBs) (nifedipine and verapamil), and statins, the patient visited the outpatient department 4 times every month. The patient underwent chest X-rays, electrocardiography, and blood tests, including cardiac enzymes, and these tests showed no significant abnormal findings.

The study was approved by the institutional review board of Samsung Medical Center (IRB approval no., 2020-09-026). The patient provided written informed consent for the publication of the clinical details and images associated with her case.

\section{Discussion}

MMD predominantly occurs in East Asian countries such as Japan and Korea. The etiology of MMD is un- 
known, and the disease mainly manifests as ischemic or hemorrhagic events [1]. In patients with MMD, histopathological changes of the pulmonary, renal, and pancreatic arteries have been reported to be similar to the stenotic changes that occur in the internal carotid arteries [2]. Rarely, coronary artery involvement in MMD can occur. Kim et al. [3] reported the case of a patient with coronary artery disease who underwent CABG and showed intimal hyperplasia intraoperatively, reflecting a common manifestation of vessels affected by MMD. The pathophysiology of vasospastic angina or coronary artery disease (CAD) among patients with MMD remains unclear, although the involvement of genetic mutations has been reported $[1,2,4]$.

Nam et al. [5] reported that 21 of 456 patients with MMD had coronary heart disease, of whom $23.8 \%$ had variant angina, which was a higher proportion than that found in the general population with CAD. Coronary artery spasms have been reported sporadically in patients with MMD. In most cases, such spasms occur without triggers, but some facilitating factors exist, such as brain surgery [6]. However, there have been no reports of intractable vasospastic events and cardiac arrest occurring after CABG surgery.

The patient in the current report had no known risk factors associated with CAD, such as diabetes mellitus, hypertension, dyslipidemia, or smoking [7]. The patient already took dual anti-platelet therapy and statins in the preoperative period. The period between diagnosis and surgery was less than 1 week, and the chance of progression of the RCA lesions was extremely low. The deduction that the patient developed an intractable vasospastic event associated with MMD is therefore quite reasonable. Additionally, in the immediate postoperative period, the patient had a relatively hypovolemic status, and the use of vasoactive agents could contribute to the situation.

The role of revascularization in vasospastic lesions remains controversial [8]. In this case, the vasospastic RCA lesion was not relieved by repeated intracoronary nitroglycerin and nicorandil injection at extremely high doses. It is possible that vasospastic obstruction and mechanical obstruction occurred simultaneously in the RCA lesion. In addition, it was obvious that the patient was hemodynamically unstable, requiring the support of veno-arterial ECMO, and might have already had ischemic insults in the myocardium. Because of time constraints, we could not wait to see the effects of CCBs or nitrates. Therefore, we and the cardiologist decided to perform stent insertion in the intractable vasospastic lesion. It would have been preferable for the patient to undergo follow-up CAG. However, because she was tired of the long hospital stay, she refused to undergo CAG and was discharged after she had improved clinically.

From a clinical point of view, active preventative measures should be taken for coronary spastic events in patients with MMD, such as perioperative administration of nitrates or CCBs. If CAD is suspected, quick evaluation, decision-making, and treatment (such as CAG or percutaneous coronary intervention) are critical. Special consideration should also be given regarding the use of vasoactive agents, such as norepinephrine, epinephrine, or vasopressin, in patients with MMD, even during postoperative management.

Although coronary artery spasms after CABG are not frequent, they are always potentially life-threatening. Therefore, it is important to keep in mind that MMD patients may be especially vulnerable to coronary spasms and to try to reduce any modifiable triggers. Further studies on the pathophysiology and treatment strategies for CAD in patients with MMD are required, as these strategies are likely to be different for MMD patients and the normal population.

\section{Conflict of interest}

No potential conflict of interest relevant to this article was reported.

\section{ORCID}

Hyeon A Kim: https://orcid.org/0000-0002-0648-948X

Young Su Kim: https://orcid.org/0000-0003-2923-3732

Wook Sung Kim: https://orcid.org/0000-0001-7808-3385

\section{References}

1. Koizumi A, Kobayashi H, Hitomi T, Harada KH, Habu T, Youssefian S. A new horizon of moyamoya disease and associated health risks explored through RNF213. Environ Health Prev Med 2016;21:55-70.

2. Bang OY, Chung JW, Kim DH, et al. Moyamoya disease and spectrums of RNF213 vasculopathy. Transl Stroke Res 2020;11:580-9.

3. Kim HJ, Jo WM, Rhu SM, Hwang JJ, Sohn YS, Choi YH. Coronary artery disease affected by moyamoya disease. Korean J Thorac Cardiovasc Surg 2002;35:231-4.

4. Guo DC, Papke CL, Tran-Fadulu V, et al. Mutations in smooth muscle alpha-actin (ACTA2) cause coronary artery disease, stroke, and Moyamoya disease, along with thoracic aortic disease. Am J Hum Genet 2009;84:617-27.

5. Nam TM, Jo KI, Yeon JY, Hong SC, Kim JS. Coronary heart disease 
in moyamoya disease: are they concomitant or coincidence? J Korean Med Sci 2015;30:470-4.

6. Choi W, Kim YN, Kim KH. Variant angina in moyamoya disease: a correlative etiology and different presentation: a case report. J Med Case Rep 2015;9:86.

7. Takaoka K, Yoshimura M, Ogawa H, et al. Comparison of the risk factors for coronary artery spasm with those for organic stenosis in a Japanese population: role of cigarette smoking. Int J Cardiol 2000; 72:121-6.

8. Tanabe Y, Itoh E, Suzuki K, et al. Limited role of coronary angioplasty and stenting in coronary spastic angina with organic stenosis. J Am Coll Cardiol 2002;39:1120-6. 